\title{
Interspecific hybridization between Brassica napus and Brassica rapa ssp. chinensis genotypes through embryo rescue and their evaluation for crossability
}

\author{
Janetta Niemann *, Magdalena Olender, Andrzej Wojciechowski, Agnieszka Tomkowiak \\ Department of Genetics and Plant Breeding, Poznań University of Life Sciences, Poznań, Poland \\ *Corresponding author: niemann@up.poznan.pl
}

\begin{abstract}
The genus Brassica contains several important crop species that are used for a variety of purposes. Brassica napus and Brassica rapa are the most important ones for their use as oilseed crops. In Brassica, interspecific hybridization is a potential and useful method for transferring valuable traits between species of commercial interest. For breeding Brassicas resistant to some diseases, i.e. clubroot, attempts of transferring resistant genes (CR) through interspecific hybridization have also been reported. The main step in the introgression process is the production of interspecific hybrids between the two species. However, the cross-incompatibility occurring in wide hybridization might hamper the possibility of obtaining hybrid progenies. For that reason, the crossability study is essential and may give an insight into the cross-compatibility relationship among the species, the direction of success of crossing, and the crossability barriers of some combinations, if any. In the present study, interspecific reciprocal crosses between $B$. napus and B. rapa ssp. chinensis genotypes were carried out in order to determine their crossability and to produce their F1 hybrids. Crossability was analyzed based on the pollen germination in$\operatorname{dex}(\mathrm{PGI})$ and the development of hybrid embryos in in vitro cultures. It was observed that the unilateral interspecific incompatibility occurred in crosses between $B$. napus $\times$ B. rapa ssp. chinensis. When $B$. napus cultivars were used as a maternal parent, pollen grains of $B$. rapa germinated well on the stigmas, while only a small number of pollen tubes could elongate near the ovules, resulting in a low development of hybrid embryos. Using the B. rapa ssp. chinensis as the pistillate parent in the crosses, it was found that the crossability was relatively higher than that in the case of the reciprocal cross. This resulted in a greater number of embryos obtained. Generally, it was concluded that the selection of parental components for hybridization is an important step for obtaining breeding success.
\end{abstract}

Key words: interspecific hybridization, crossability, embryo rescue, Brassicas, clubroot resistance

\section{Introduction}

Many Brassica species and allies are wild and weedy with useful genes which could be incorporated into breeding programs, including research into the cytoplasmic and nuclear male sterility; the resistance to diseases; insect or nematode pests; and the tolerance of cold, salt, and drought conditions (Chen et al., 2011). Thus, interspecific hybridization is still a useful approach for the introgression of these favorable agronomic traits from wild species into cultivated crops (Mei et al., 2010; Niemann et al., 2012, 2014). It is interesting, mainly because of the growing yield losses in oilseed rape caused by various pathogens among which one of the most dangerous is Plasmodiophora brassicae (Pageau et al., 2006). For breeding Brassicas resistant to clubroot, attempts of transferring CR through interspecific hybridization have been reported (Gowers, 1982; Diederichsen et al., 2009; Niemann et al., 2015). Among the two progenitor species of Brassica napus, clubroot resistance is more frequently found in turnips (Brassica rapa; A genome; $n=10$ ) (Hirai, 2006). This is why the crosses between $B$. napus and the three genotypes of $B$. rapa ssp. chinensis with potentially increased resistance to clubroot were made. However, interspecific hybridization between allotetraploid and diploid Brassica species is difficult; as a result, cross-incompatibility often hampers the production of those hybrid progenies. While most attempts on interspecific hybridization were based on conventional techniques (hand pollination), the frequency of hybrids was low. In addition, hybrids were obtained at relatively higher 
frequency when $B$. rapa was used as a female parent, indicating a strong incompatibility in the reverse cross (Ammitzboll et al., 2005). Therefore, the main goal of our study was to evaluate the crossability between B. napus and B. rapa ssp. chinensis genotypes and to obtain $\mathrm{F} 1$ hybrids between those genotypes using an embryo rescue technique.

\section{Materials and methods}

\section{Plant materials and crosses}

Five $B$. napus seed cultivars, i.e. Jet Neuf, Lisek, Skrzeszowicki, Californium, and Zhongshuang 9, were selected from the resources of the Genetics and Plant Breeding Department, Poznań University of Life Sciences, while seeds of $B$. rapa accessions (A: Chinense Cabbage PI430485 98CI; B: Pak Choi 08 007569; C: Chinense Cabbage 08 006169) came from the Warwick HRI Genetic Resources Unit. The reciprocal crosses were carried out in the glasshouse of Genetics and Plant Breeding Department during spring 2013. Each genotype was represented by four to six plants. The pollen of pollinators was placed on stigmas immediately after emasculation, which was done at the closed bud stage. In each cross-combination, around 11 to 90 flower buds were pollinated.

Pollinated pistils were divided into two parts. Half of them were allocated for the microscopic examinations. The rest of pistils were left on the plants to silique formation.

\section{The evaluation of crossability based on the PGI}

Observations of pollen grain germination and pollen tube (PT) growth were conducted after cross-pollination (CP) of the chosen Brassica genotypes. For this purpose, pistils of three $B$. napus cultivars i.e. Jet Neuf, Californium, Skrzeszowicki, and one B. rapa ssp. chinensis genotype (08 006169) were collected $48 \mathrm{~h}$ after pollination. They were next fixed and stained with aniline blue (Antkowiak and Wojciechowski, 2006). By applying a fluorescent microscopy technique, the assessment of pollen tube growth was made. Six pistils were analyzed in each combination. The intensity of pollen grain germination and pollen tube penetration was expressed by the six degree scale (Niemann et al., 2014), where 0 was the absence of PT, 1-4 was the intermediate number of PT, and 5 was the largest number of PT. The crossability
(CC: cross-compatibility; CI: cross-incompatibility) was evaluated on the basis of the PGI according to Kaneko et al. (2009); and also, pistils were classified into five specimens: 1 ) the number of pistils with pollen grains, 2) the number of pistils in which pollen grains do not germinate, 3) the number of pistils in which pollen grains germinate on the stigmas, 4 ) the number of pistils in which pollen tubes enter the style tissue, and 5) the number of pistils in which pollen tubes penetrate close to or enter the ovules.

The value of PGI was obtained from the formula of $P G I=(1 b+2 c+3 d+4 e) /(a+b+c+d+e)$.

In the case of PGI equal or higher than 2, it was concluded that there existed compatibility.

\section{The effectiveness of crosses}

All hybridizations were performed with the application of an in vitro culture of isolated embryos according to the method described by Wojciechowski (1998).

The immature embryos were isolated from young siliques at different developmental stages, i.e. heart, early torpedo, torpedo, late torpedo, or nearly matured embryos, 14-19 days after pollination. For the embryo culture, basal White (W, 1963), Murashige and Skoog (MS, 1962), Murashige and Skoog modified by Keller and Armstrong $\left(\mathrm{Ms}_{\mathrm{k}}, 1977\right)$, and Nitsh and Nitsh $\left(\mathrm{H}_{3}\right.$, 1969) media were applied (Table 1 ).

The effectiveness of an interspecific hybridization of $B$. napus with chosen $B$. rapa genotypes was expressed in three different ways: by the number of embryos obtained to the mean number of well-developed ovules, by the fertility (siliqua/pollinated flowers (\%) - Figure 1), and by the number of plants regenerated in the soil.

\section{Results}

\section{Evaluation of crossability based on the PGI}

Generally, a different intensity of pollen tube growth and the differences at PGI value were observed in most cross-combinations, depending on which form was used as a maternal parent. It was observed that after crosspollination of $B$. napus cultivars with $B$. rapa ssp. chinensis pollen grains, the intensity of pollen tube growth was low, and the pollen tubes were observed mainly in the style or in the ovary. Occasionally, a low number of ovules were penetrated by pollen tubes (PGI value less than 2) - Figure $2 \mathrm{E}$. The crossing between three B. na- 
Table 1. Pattern of Brassica embryos cultures in in vitro conditions

\begin{tabular}{|c|c|c|c|c|}
\hline No. & Media & Embryo/plant developmental stage & $\begin{array}{l}\text { In vitro } \\
\text { culture } \\
\text { duration }\end{array}$ & $\begin{array}{c}\text { Conditions } \\
\text { of in vitro } \\
\text { cultures }\end{array}$ \\
\hline 1. & White & $\mathrm{H}^{*}, \mathrm{ET}$ & 7 days & \multirow{5}{*}{$\begin{array}{l}\text { Growth } \\
\text { room** }\end{array}$} \\
\hline 2. & Murashige \& Skoog & T, LT, NM & 3-4 weeks & \\
\hline 3. & $\begin{array}{l}\text { Murashige \& Skoog } \\
\text { in Keller modification }\end{array}$ & explants developing callus and first leaves & 5-11 weeks & \\
\hline 4. & Nitsch \& Nitsch & seedlings & 2 weeks & \\
\hline \multirow{2}{*}{5.} & \multirow{2}{*}{ Soil } & \multirow{2}{*}{ well rooted seedlings } & 7 days & \\
\hline & & & until matured plants & greenhouse \\
\hline
\end{tabular}

${ }^{*} \mathrm{H}$ - heart, ET - early torpedo stage, T - torpedo stage, LT - late torpedo, NM - nearly matured embryo,

** conditions: temperature $26^{\circ} \mathrm{C}$; photoperiod: $16 \mathrm{~h}$ light, $8 \mathrm{~h}$ dark

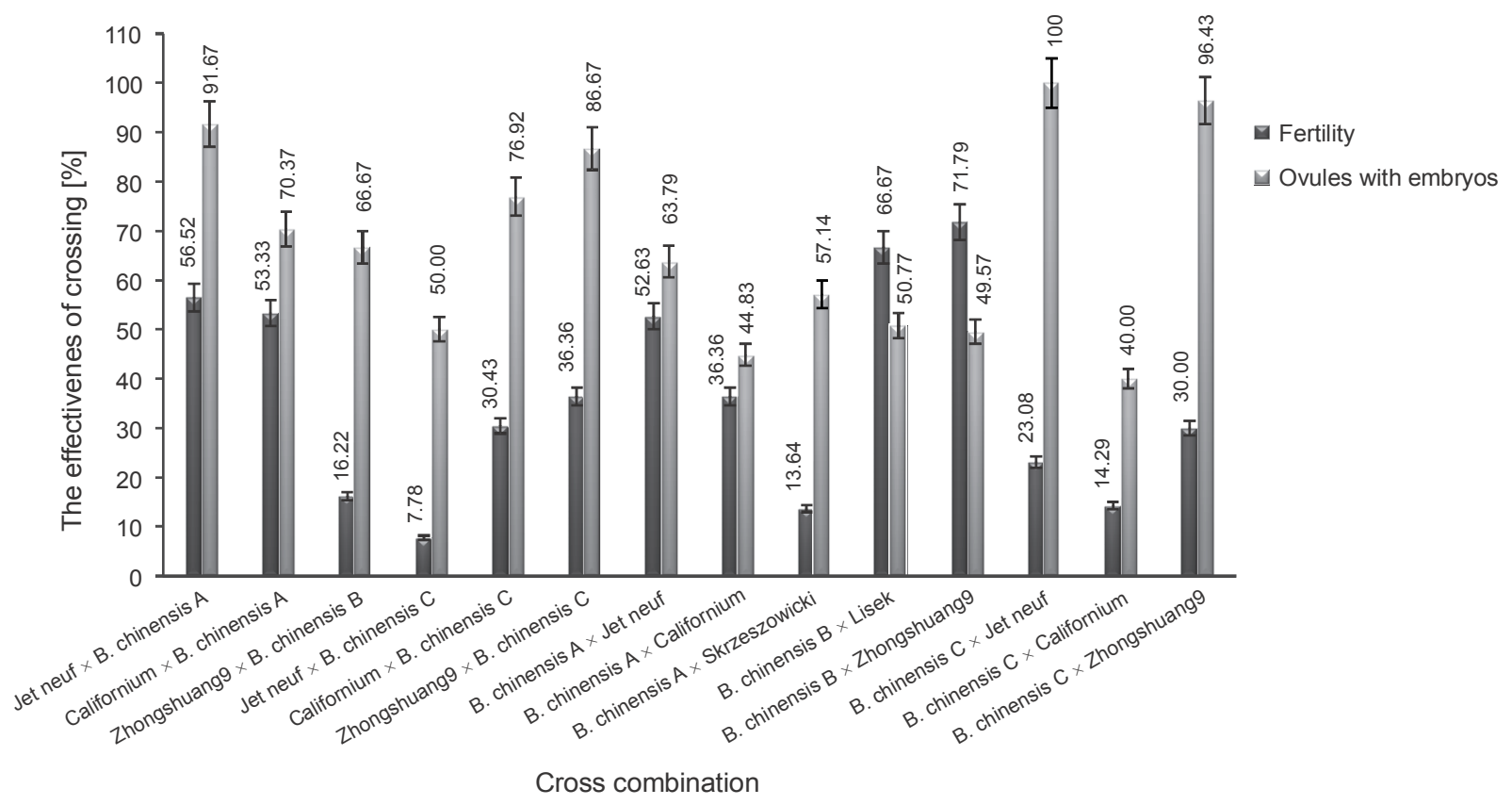

Fig. 1. Effectiveness of reciprocal crosses between $B$. napus cultivars with $B$. rapa ssp. chinensis genotypes expressed by the fertility and ovules with embryos (\%). Error bars indicate within subject standard error

pus cultivars and the tested genotype of B. rapassp. chinensis showed that these two species do not suit each other concerning their compatibility, and in these cases, there are some prezygotic incompatibility barriers. Quite a different situation was observed in crosses in which $B$. rapa ssp. chinensis plants were used as a maternal and $B$. napus cultivars as a pollen donor. In those cases, the intensity of pollen tube growth was higher, and PGI ranged from 2.11 (B. rapa ssp. chinensis $\times$ B. napus cv. Californium) to 2.69 ( $B$. rapa ssp. Chinensis $\times B$. napus $\mathrm{cv}$. Jet Neuf) with a mean value of 2.32 (Table 2, Fig. 2A-D).

\section{The effectiveness of crosses}

The highest fertility was observed in crosses between B. rapa ssp. chinensis B and B. napus cv. Lisek and Zhongshuang 9. It was $66.67 \%$ and $71.79 \%$, respectively (Fig. 1). Whereas in one of the reverse crossing, i.e. $B$. napus cv. Lisek $\times B$. rapa ssp. chinensis $\mathrm{B}$, there were no siliques obtained at all. Similarly, no siliques were received in $B$. napus cv. Lisek $\times B$. rapa ssp. chinensis $\mathrm{C}$ and $B$. napus cv. Skrzeszowicki $\times$ B. rapa ssp. chinensis A. Moreover, in all cross-combinations, quite a high percentage of ovules with embryos were obtained 
Table 2. Pollen germination index (PGI) in reciprocal crosses of chosen Brassica genotypes

\begin{tabular}{|c|c|c|}
\hline \multicolumn{2}{|c|}{ Combination of pollination } & $\begin{array}{l}\text { PGI } 48 \mathrm{~h} \text { after } \\
\text { pollination }\end{array}$ \\
\hline B. napus cv. Jet Neuf & \multirow{3}{*}{ B. rapa ssp. chinensis $\mathrm{C}$} & 1.94 \\
\hline B. napus cv. Californium & & 1.81 \\
\hline B. napus cv. Skrzeszowicki & & 1.88 \\
\hline \multicolumn{2}{|c|}{ Mean } & $1.88 \pm 0.06^{*}$ \\
\hline \multirow{3}{*}{ B. rapa ssp. chinensis $\mathrm{C}$} & B. napus cv. Jet Neuf & 2.69 \\
\hline & B. napus cv. Californium & 2.11 \\
\hline & B. napus cv. Skrzeszowicki & 2.17 \\
\hline \multicolumn{2}{|c|}{ Mean } & $2.32 \pm 0.32$ \\
\hline
\end{tabular}

* Mean \pm SD

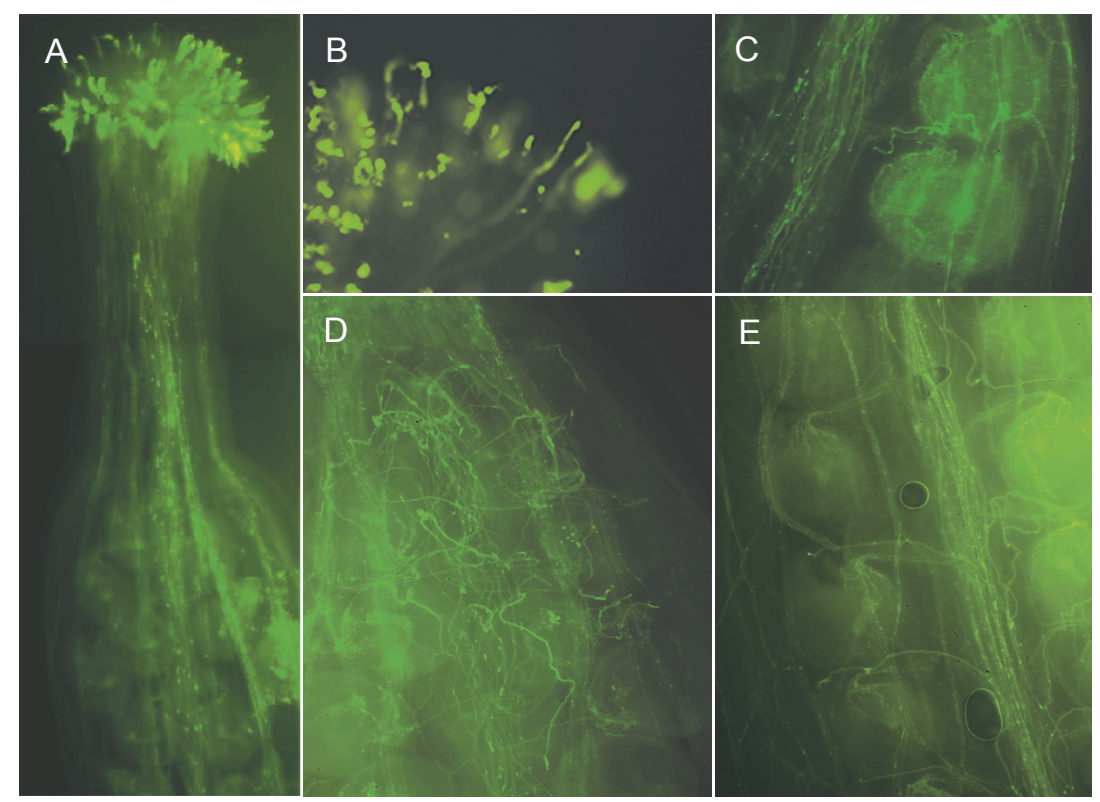

Fig. 2. Pollen germination and pollen tube elongation in pistils of analyzed Brassica genotypes: A) The pistil of $B$. rapa ssp. chinensis A after pollination with $B$. napus cv. Californium; B) Pollen grains germinating on the stigma, B. napus cv. Zhongshuang $9 \times B$. rapa ssp. chinensis A; C-D) pollen tubes in the ovary, B. rapa ssp. chinensis A $\times$ B. napus Jet Neuf; E) Pollen tube penetrating the ovule, $B$. napus cv. Lisek $\times$ B. rapa ssp. chinensis A

ranging from $40 \%$ in $B$. rapa ssp. chinensis $\mathrm{C} \times B$. napus cv. Californium to $96.43 \%$ in $B$. rapa ssp. chinensis C $\times B$. napus cv. Zhongshuang 9 . In the case of the lastmentioned cross-combination, plants were obtained from $51.85 \%$ of the isolated embryos.

In this experiment, from 236 pollinated flowers, up to 452 embryos (Fig. 3A) were received after crosses between all $B$. rapa ssp. chinensis $\times B$. napus genotypes. In the case of reverse hybridization, a lower number of embryos were formed out only 80 , despite the pollination of a greater number of flowers (273). However, the efficiency of embryo rescue presented as the ratio of the regenerated plants to the isolated embryos was higher in these variants of crosses, and it was $73.7 \%$ (B. napus $\times B$. rapa ssp. chinensis), while only $35 \%$ of plants were regenerated from the $B$. rapa ssp. chinensis $\times B$. napus crosses. Generally, in all tested cross-combinations, 509 flowers were pollinated and 220 plants were regenerated through embryo cultures, wherein most plants were obtained from the crosses between $B$. rapa ssp. chinensis $\times B$. napus genotypes -161 (Fig. 3B-F, Table 3). In those cross-combinations, the highest number of em- 
Table 3. Effectiveness of interspecific crosses between B. napus cultivars and B. rapa ssp. chinensis genotypes expressed by the number of regenerated plants in the soil

\begin{tabular}{|c|c|c|c|c|c|c|c|c|c|c|c|}
\hline \multicolumn{4}{|c|}{ Cross-combination } & \multirow{3}{*}{$\begin{array}{c}\text { No. } \\
\text { of pollinated } \\
\text { flowers }\end{array}$} & \multirow{3}{*}{$\begin{array}{c}\text { No. } \\
\text { of siliques }\end{array}$} & \multicolumn{4}{|c|}{ No. of isolated embryos } & \multirow{3}{*}{$\begin{array}{l}\text { No. of plant } \\
\text { rooted in } \mathrm{H}_{3}\end{array}$} & \multirow{3}{*}{$\begin{array}{l}\text { No. of plant } \\
\text { in soil }\end{array}$} \\
\hline \multirow{2}{*}{\multicolumn{2}{|c|}{ 우 }} & \multirow{2}{*}{\multicolumn{2}{|c|}{$\sigma^{x}$}} & & & \multirow{2}{*}{ Total } & \multicolumn{3}{|c|}{ In different developmental stages } & & \\
\hline & & & & & & & $\mathrm{H}$ & ET & LT & & \\
\hline \multirow{9}{*}{ B. napus } & Jet Neuf & \multirow{3}{*}{\multicolumn{2}{|c|}{ B. rapa ssp. chinensis A }} & 23 & 13 & 11 & 0 & 0 & 11 & 7 & 7 \\
\hline & Californium & & & 15 & 8 & 19 & 0 & 8 & 11 & 12 & 12 \\
\hline & Skrzeszowicki & & & 13 & 0 & 0 & 0 & 0 & 0 & 0 & 0 \\
\hline & Lisek & \multirow{2}{*}{\multicolumn{2}{|c|}{ B. rapa ssp. chinensis B }} & 11 & 0 & 0 & 0 & 0 & 0 & 0 & 0 \\
\hline & Zhongshuang9 & & & 37 & 6 & 2 & 0 & 0 & 2 & 0 & 0 \\
\hline & Jet Neuf & \multirow{4}{*}{\multicolumn{2}{|c|}{ B. rapa ssp. chinensis C }} & 90 & 7 & 2 & 0 & 0 & 2 & 2 & 2 \\
\hline & Lisek & & & 28 & 0 & 0 & 0 & 0 & 0 & 0 & 0 \\
\hline & Californium & & & 23 & 7 & 20 & 0 & 7 & 13 & 19 & 18 \\
\hline & Zhongshuang9 & & & 33 & 12 & 26 & 0 & 18 & 8 & 26 & 20 \\
\hline \multicolumn{4}{|c|}{ Total } & 273 & 53 & 80 & 0 & 33 & 47 & 66 & 59 \\
\hline \multirow{3}{*}{\multicolumn{2}{|c|}{ B. rapa ssp. chinensis A }} & \multirow{8}{*}{ B. napus } & Jet Neuf & 19 & 10 & 74 & 24 & 50 & 0 & 22 & 22 \\
\hline & & & Californium & 22 & 8 & 39 & 20 & 19 & 0 & 10 & 8 \\
\hline & & & Skrzeszowicki & 66 & 9 & 68 & 12 & 50 & 6 & 60 & 57 \\
\hline \multirow{2}{*}{\multicolumn{2}{|c|}{ B. rapa ssp. chinensis B }} & & Lisek & 15 & 10 & 66 & 29 & 35 & 2 & 0 & 0 \\
\hline & & & Zhongshuang9 & 39 & 28 & 173 & 64 & 80 & 29 & 61 & 59 \\
\hline \multirow{3}{*}{\multicolumn{2}{|c|}{ B. rapa ssp. chinensis C }} & & Jet Neuf & 13 & 3 & 1 & 0 & 1 & 0 & 1 & 1 \\
\hline & & & Californium & 42 & 6 & 4 & 1 & 3 & 0 & 0 & 0 \\
\hline & & & Zhongshuang9 & 20 & 6 & 27 & 0 & 0 & 27 & 14 & 14 \\
\hline \multicolumn{4}{|c|}{ Total } & 236 & 80 & 452 & 150 & 238 & 64 & 168 & 161 \\
\hline \multicolumn{4}{|c|}{ General } & 509 & 133 & 532 & 150 & 271 & 111 & 234 & 220 \\
\hline
\end{tabular}



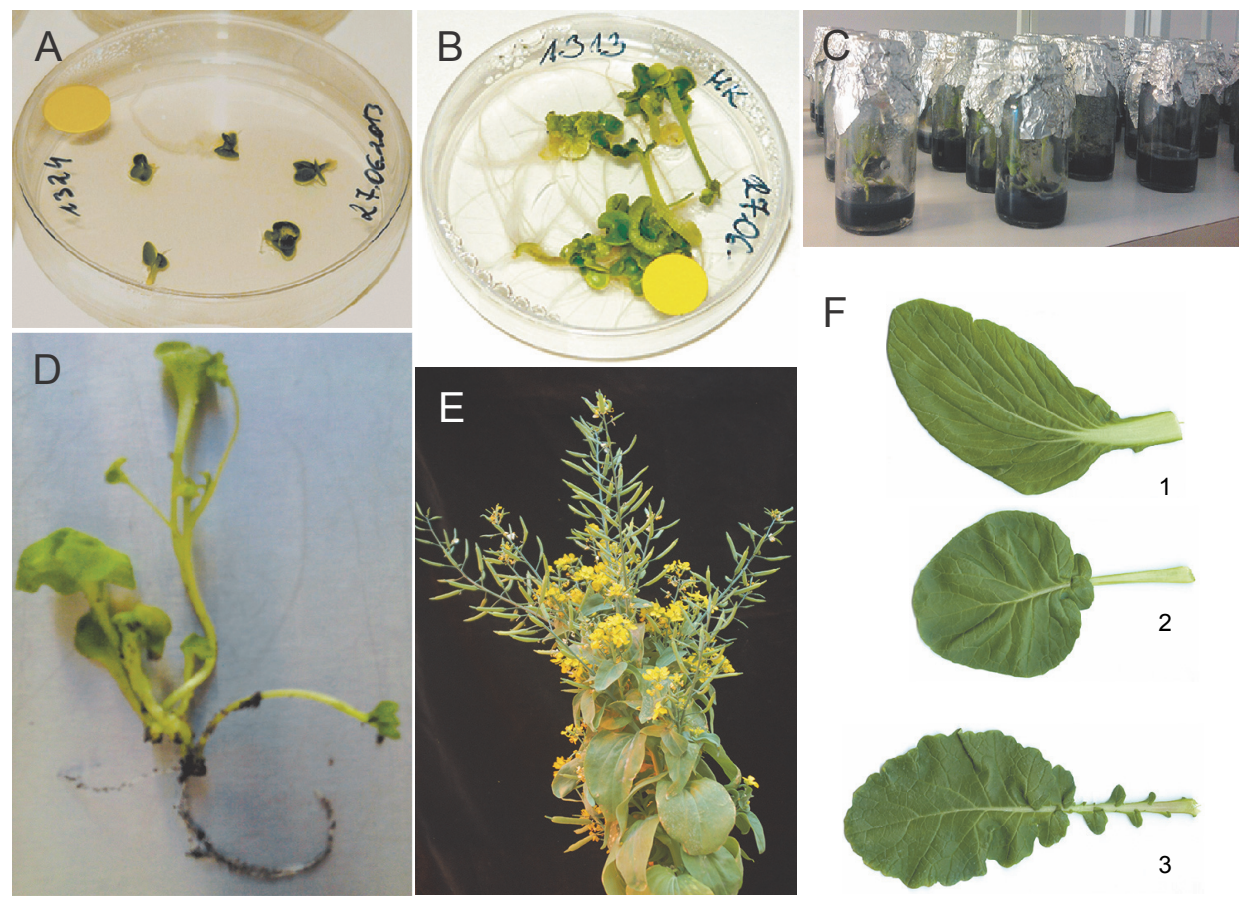

Fig. 3. Regeneration of plants in in vitro embryo cultures after crossing B. rapa ssp. chinensis A $\times$ B. napus cv. Jet Neuf: A) Fully matured embryos on MS medium; B) Regenerated seedlings on $\mathrm{MS}_{\mathrm{k}}$ medium; C) Regenerated plant on rooting $\mathrm{H}_{3}$ medium; D) Regenerated plant just before being transferred to soil; E) Regenerated plant in the soil; F) Comparative leaf morphology of F1 hybrid plants: 1 - B. rapa, 2 - F1 hybrids, 3 - B. napus

bryos was isolated at the heart and early torpedo stages, while after $B$. napus $\times$ B. rapa crosses, no embryos have been obtained at the heart developmental stage.

\section{Discussion}

To date, successful attempts of hybridization between Brassica rapa and Brassica napus have been published in numerous scientific papers (Fitz et al., 2007; Choudhary et al., 2012). Crossability between B. napus and $B$. rapa has also been examined, specifically in terms of the genes introgression from $B$. napus to $B$. rapa, which is mainly considered a weed in America and Europe (Tsuda et al., 2014). However, to the best of our knowledge, this is one of the first studies focusing directly on the potential of hybrid production and crossability between diploid B. rapa ssp. chinensis genotypes and allotetraploid $B$. napus in terms of the portability of the resistance genes. It is known that in $B$. rapa, a high level of genetic diversity was observed, and moreover, species of $B$. rapa vary in their level of cross-compatibility (Olsson, 1960).

Thus, it is difficult to compare our results with those presented in literature, particularly because $B$. rapa is a species consisting of various widely cultivated subspecies including pekinensis, chinensis, the turnip and the turnip rape (B. rapa subsp. oleifera). Within the species, different genotypes may differ in their propensity to cross with other species depending on their crossability or self-incompatibility. For this reason, experimental hybridization studies for plant breeding often incorporate diverse genotypes. This means that even if one genotype crosses with a wild relative, others within the same species may not. Although, according to Downey et al. (1980), B. rapa varieties are generally self-incompatible (Indian brown sarson and toria types as well as North American and European B. rapa cultivars), some of them are highly self-compatible (Indian yellow sarson form of $B$. rapa).

Despite the fact that $B$. rapa is one of the most comprehensively studied crops, as with $B$. napus, relatively few studies report success of hybrid production quantitatively (Fitz et al., 2007).

It is commonly known that canola (B. napus, AACC genome) and $B$. rapa (AA genome) have a common set of chromosomes, which facilitates interspecific gene flow between these two species. Spontaneous hybridization 
in the field is possible, and natural B. napus $\times B$. rapa hybrids have been reported in several countries, including Canada (Yoshimura et al., 2006), Denmark (Hansen et al., 2001), and the United Kingdom (Allainguillaume et al., 2006). In field trials, under natural conditions, the success of hybridization varies widely, depending on the experimental design and the direction of crosses (Pallett et al., 2006). However, subsequent introgression of alleles from $B$. napus to B. rapa occurs infrequently (Leflon et al., 2006). In the laboratory experiments, $B$. rapa pollen has a significantly lower degree of fitness on $B$. napus than conspecific pollen, and hybrid zygote survival is markedly reduced in comparison to conspecific zygotes (Hauser et al., 1997). In addition, B. rapa $\times$ B. napus hybrids have been found to have reduced fertility and lower seed set compared to either parental species (Jorgensen and Andersen, 1994).

The results of our investigation showed that the biggest number of embryos was received when $B$. rapa ssp. chinensis was treated as a female parent. These results are consisted with those obtained from the literature data (Ammitzbol et al., 2005) because they demonstrate that hybridization rates are significantly higher if $B$. rapa is a female parent, rather than vice versa, due to the self-incompatibility of $B$. rapa.

Our results confirmed that there is unilateral crossability between $B$. rapa ssp. chinensis and B. napus genotypes. When $B$. rapa ssp. chinensis was a female parent and $B$. napus was a male parent in all cases, crosscompatibility was observed with a mean PGI value $=2.32 \pm 0.26$. In reciprocal crosses, generally there was incompatibility ( $\mathrm{PGI}=1.87 \pm 0.05^{*}$ ). According to Long et al. (1992), pekinensis group was more compatible with Brassino raphanus than rapifera group in B. rapa. In addition, kales were even more compatible than other varieties of $B$. oleracea, which suggests that the selection of certain species, subspecies, varieties or cultivars as pollen parents is important and may improve the cross-compatibility between them. Although, we obtained hybrid plants in both directions of crosses $B$. rapa ssp. chinensis $\times B$. napus and $B$. napus $\times B$. rapa ssp. chinensis (161 and 59, respectively). A greater number of regenerated plants were obtained when $B$. rapa ssp. chinensis was a female parent. Those results are coinciding with our observation of PGI value. This is particularly interesting in view of our previous studies on crossability in different Brassica species (Niemann et al., 2014). In those cases, crosses between self-compatible (SC) cultivar Californium (B. napus) and self-incompatible (SI) B. rapa ssp. pekinensis were successful only when SC cultivar was used as a maternal form. Reciprocal crosses $(\mathrm{SI} \times \mathrm{SC})$ failed. Fitz et al. (2007) made a similar observation related to the crossability barriers. Crosses between B. napus and B. rapa (a progenitor of $B$. napus) were very successful with a median rate of 2.29 hybrids/pollination when $B$. napus was the female parent in the cross but lower ( 0.44 hybrids/pollination) when $B$. napus was the male parent in the cross. In both directions, the rates of production ranged widely across trials (Fitz et al., 2007). The same authors proved that crosses between $B$. napus and $B$. rapa were more successful when the amphidiploid species, i.e. B. napus, was used as a female parent. Reciprocal crosses were generally unsuccessful. Moreover, Choudhary et al. (2012) demonstrated that amongst crosses involving three varieties/forms of $B$. rapa with $B$. napus, yellow sarson produced a maximum number of hybrids (18.0\%) followed by brown sarson (11.3\%) and toria (6.9\%). Thus, the success rate of cross-fertility of three varieties of $B$. rapa with $B$. napus was in the order yellow sarson $>$ brown sarson $>$ toria. These observations are similar to our results indicating that the success of interspecific crosses depends not only on the species but also on the form/varieties of the species involved. That is why we observed differences in the number of $B$. napus $\times$ B. rapa ssp. chinensis hybrids obtained through embryo rescue. The latest was probably related to interspecific crossability between genotypes.

\section{Conclusions}

1) Observations of the pollen tube growth and embryo set indicate unilateral interspecific compatibility in the crosses where B. rapa ssp. chinensis was used as a maternal form. However, the number of obtained embryos depended on the genotype.

2) The highest number of hybrids was obtained from crosses between $B$. rapa ssp. chinensis $\times B$. napus.

3) The degree of hybridization between $B$. rapa and $B$. napus varied depending on subspecies of $B$. rapa, which were used in the crosses and the direction of crossing.

\section{Acknowledgements}

The Authors thanks to the Ministry of Agriculture and Rural Development, Poland for financial support of task no. 54 . 


\section{References}

Allainguillaume J., Alexander M., Bullock J.M. (2006) Fitness of hybrids between rapeseed (Brassica napus) and wild Brassica rapa in natural habitats. Molec. Ecol.15: 1175-1184.

Ammitzboll H., Mikkelsen T.N., Jorgensen R.B. (2005) Transgene expression and fitness of hybrids between oilseed rape and Brassica rapa. Environ. Biosaf. Res. 4(1): 3-12.

Antkowiak W., Wojciechowski A. (2006) The evaluation of selfcompatibility and crossability in the genus Pyrus based on the observation of pollen tubes growth. Acta Agrobot. 59(1): 91-97.

Chen L.P., Ge X.H., Yao X.Ch., Feng Y.H., Li Z.Y. (2011) Synthesis and characterization of interspecific trigenomic hybrids and allohexaploids between three cultivated Brassica allotetraploids and wild species Brassica fruticulosa. Afr. J. Biotechnol. 10(57): 12171-12176.

Choudhary B.R., Joshi P. (2012) Cytomorphology of Brassica napus $x$ B. rapa hybrids and patterns of variation in the F-2 derivatives. Caryologia 65: 316-321.

Diederichsen E., Frauen M., Linders E.G.A., Hatakeyama K., Hirai M. (2009) Status and perspectives of clubroot resistance breeding in crucifer crops. J. Plant Growth Regul. 28: 265-281.

Downey R.K., Klassen A.J., Stringam G.P. (1980) Rapeseed and mustard. In: Hybridization of Crop Plants. Am. Soc. Crop. Sci.: 495-509.

Fitz J.T., Armstrong T., Newstrom-Lloyd L., Wilton A., Cochrane M. (2007) Hybridisation within Brassica and allied genera: evaluation of potential for transgene escape. Euphytica 158(1-2): 209-230.

Gowers S. (1982) The transfer of characters from Brassica campestris L. to Brassica napus L.: Production of clubroot-resistant oil-seed rape (B. napus ssp. oleifera). Euphytica 31: 971-976.

Hansen L.B., Siegismund H.R., Jorgensen R.B. (2001) Introgression between oilseed rape (Brassica napus L.) and its weedy relative $B$. rapa $L$. in a natural population. Gen. Res. Crop. Evol. 48: 621-627.

Hauser T.P., Jørgensen R.B., Østergård H. (1997) Preferential exclusion of hybrids in mixed pollinations between oilseed rape (Brassica napus) and weedy B. campestris (Brassicaceae). Am. J. Bot. 84: 756-762.

Hirai M. (2006) Genetic analysis of clubroot resistance in Brassica rapa. Breed. Sci. 56: 223-229.

Jorgensen R.B., Andersen B. (1994) Spontaneous hybridization between oilseed rape (Brassica napus) and weedy Brassica campestris (Brassicaceae). A risk of growing genetically modified oilseed rape. Am. J. Bot. 81: 1620-1626.

Kaneko Y., Bang S.W., Matsuzawa Y. (2009) Distant hybridization. In: Biology and Breeding of Crucifers. Ed. Gupta S.K. New York, Taylor \& Francis Group: 207-247.

Keller W.A., Armstrong K.C. (1977) Embryogenesis and plant regeneration in Brassica napus anther culture. Can. J. Bot. 55(10): 1383-1388.
Leflon M., Eber F., Letanneur J. C., Chelysheva L., Coriton O., Huteau V., Ryder C. D., Barker G., Jenczewski E., Chevre A.M. (2006) Pairing and recombination at meiosis of Brassica rapa $(A A)_{x}$ Brassica napus $(A A C C)$ hybrids. Theor. Appl. Genet. 113: 1467-1480.

Long M.H., Xing G.M., Okubo H., Fujieda K. (1992) Cross compatibility between Brassicoraphanus and cruciferous crops, and rescuing the hybrid embryos through ovary and embryo cultures. J. Fat. Agr., Kyushu Univ. 37: 29-39.

Mei J., Li Q., Yang X. (2010) Genomic relationships between wild and cultivated Brassica oleracea L. with emphasis on the origination of cultivated crops. Gen. Res. Crop Evol. 57: 687-692.

Murashige T., Skoog F. (1962) A revised medium for rapid growth and bio-assays with tobaco tissue culture. Physiol. Plant. 15: 473-497.

Niemann J., Wojciechowski A., Janowicz J. (2012) Broadening the variability of quality traits in rapeseed through interspecific hybridization with application of immature embryo culture. BioTechnologia, 93(2): 109-115.

Niemann J., Kotlarski Sz., Wojciechowski A. (2014) The evaluation of self-incompatibility and crossability in choosen Brassica species based on the observation of pollen tubes growth and seed set. Acta Sci. Pol., Agricult. 13(1): 51-59.

Niemann J., Kaczmarek J., Wojciechowski A., Olejniczak J., Jędryczka M. (2015) Hybrids within the genus Brassica and chemical mutants of Brassica napus - the potential sources of resistance to clubroot (Plasmodiophora brassicae). Prog. Plant Prot. (Post. Ochr. Roślin) 55(1): 87-91.

Nitsh J.P., Nitsh C., Hamon S. (1969) Production de Nicotiana tabacum diploids a partir de cals haploides cultives in vitro. C.R. Acad. Sci. Paris 269: 1275-1278.

Olsson G. (1960) Species crosses within the genus Brassica. II. Artifical Brassica napus L. Hereditas. 46(3-4): 351-386.

Pageau D., Lajeunesse J., Lafond J. (2006) Impact de l'hernie des cruciferes (Plasmodiophora brassicae) sur la productivite et la qualite du canola. Can. J. Plant Path. 28: 137-143.

Pallett D.W., Huang L., Cooper J.I., Wang H. (2006) Within-population variation in hybridisation and transgene transfer between wild Brassica rapa and Brassica napus in the UK. Ann. Appl. Biol. 148: 147-155.

Tsuda M., Ohsawa R., Tabei Y. (2014) Possibilities of direct introgression from Brassica napus to $B$. juncea and indirect introgression from $B$. napus to related Brassicaceae through B. juncea. Breed. Sci. 64(1): 74-82.

White P.R. (1963) The cultivation of animal and plant cells. New York, The Ronald Press.

Wojciechowski A. (1998) Regeneration abilities of chosen genotypes of Brassica in in vitro cultures. Ann. Agricult. Univ. Poznan 289: 1-58.

Yoshimura Y., Beckie H.J., Matsuo K. (2006) Transgenic oilseed rape along transportation routes and port of Vancouver in western Canada. Environ. Biosafety Res. 5: 67-75. 